\title{
Opposing effects of IL-10
}

Children with acute or recent malarial infection are particularly vulnerable to bacteraemia caused by non-typhoidal Salmonella species. Now, Lokken et al. show that interleukin-10 (IL-10) - which is produced in response to malaria infection - increases susceptibility to non-typhoidal Salmonella infection by suppressing the ability of macrophages and neutrophils to control the bacterial infection.

To investigate the immunological mechanisms by which malaria contributes to this increased risk of infection, the authors co-infected mice with the malarial parasite Plasmodium yoelii and Salmonella enterica subsp. enterica serovar Typhimurium. Co-infection had no effect on the levels of parasite infection. However, co-infected mice showed increased bacterial loads in the liver at day 2 , and at day 4 these mice had a high bacterial burden in the blood compared with mice that were infected with $S$. Typhimurium alone.

\section{ce}

co-infected

mice and

mice that

were infected with $P$. yoelii alone had higher levels of circulating IL-10

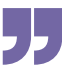

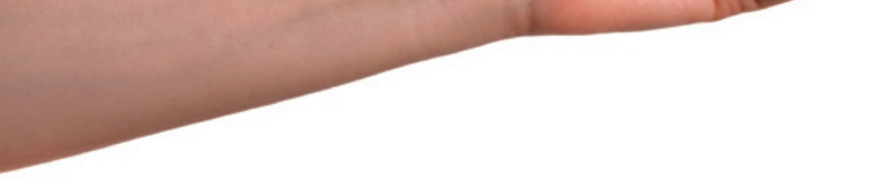

Next, the authors carried out histopathological analyses to investigate whether underlying malaria infection affected inflammatory responses in the livers of mice that were infected with $S$. Typhimurium. Interestingly, co-infected mice had fewer microabscesses and smallersized lesions than mice that were infected with $S$. Typhimurium alone. This indicates that a reduced inflammatory response to invasive $S$. Typhimurium in the liver during co-infection with malaria could contribute to the increased bacterial burden in this tissue.

IL-10 production by $\mathrm{CD} 4^{+} \mathrm{T}$ cells has previously been shown to have a protective role during malaria infection by preventing excessive pathology both in mouse models and in patients with malaria. The authors found that co-infected mice and mice that were infected with $P$. yoelii alone had higher levels of circulating IL-10 than mice infected with only $S$. Typhimurium. Neutralizing antibodies against IL-10 reduced bacterial burdens in both the liver and blood of co-infected mice but not in mice that were infected with $S$. Typhimurium alone. Furthermore, co-infected mice that lacked Il10 did not show reduced expression of genes that encode pro-inflammatory cytokines compared with control mice.
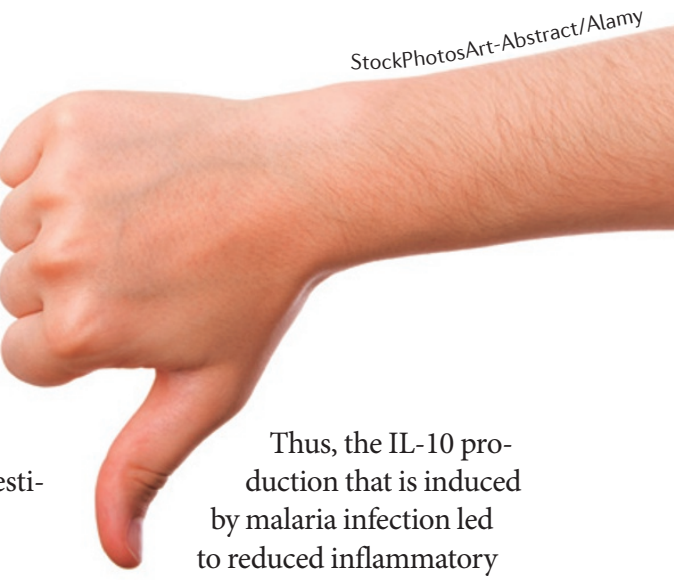

Thus, 10 production that is induced by malaria infection led to reduced inflammatory responses to $S$. Typhimurium in the liver.

In a mouse model of chronic typhoid, $S$. Typhimurium persists within alternatively activated macrophages. Hence, the authors investigated whether IL-10 production by macrophages could have a role in the malaria-mediated susceptibility to $S$. Typhimurium infection. Indeed, they found that macrophages are both the major producers of and the targets of the IL-10 that exacerbates systemic bacterial infection in co-infected mice.

Taken together, the results of this study indicate that IL-10 has opposing effects during coinfection with malaria parasites and $S$. Typhimurium - it protects against the excessive inflammation caused by malaria infection but it also leads to increased susceptibility to $S$. Typhimurium. It will be interesting to see whether this holds true in humans with malaria.

Elisabeth Kugelberg

ORIGINAL RESEARCH PAPER Lokken, K. L. et al. Malaria parasite infection compromises control of concurrent systemic non-typhoidal Salmonella infection via IL-10-mediated alteration of myeloid cell function. PLoS Pathog. 10, e1004049 (2014) 